\title{
NeuroTracker Three-Dimensional Multiple Object Tracking (3D- MOT): A Tool to Improve Concentration and Game Performance among Basketball Athletes
}

\author{
${ }^{1}$ Komarudin Komarudin*, ${ }^{1}$ Mulyana Mulyana, ${ }^{1}$ Berliana Berliana, ${ }^{1}$ Ira Purnamasari \\ ${ }^{1}$ Department of Sport Coaching Education, Faculty of Sport and Health Education, Universitas Pendidikan \\ Indonesia, Bandung, Indonesia.
}

Submitted 01 October 2020; Accepted in final form 28 November 2020.

\begin{abstract}
Background. Basketball is a dynamic sport where athletes are expected to observe the fast movements of team players and opponents in a performance. This condition requires a collective focus on the stipulated tasks, to achieve peak performance. Besides, training is frequently performed to improve concentration and athlete performance. This instigates the need for technology-based methods, including the NeuroTracker 3D MOT. Objectives. This study aims to examine differences in the effect of NeuroTracker 3D MOT and conventional training on the concentration and game performance of basketball athletes. Methods. This experimental study involved a pre and post-training session design, and the treatments were provided to 21 university basketball athletes. Furthermore, a total of 12 NeuroTracker 3D MOT training were performed by the experimental group, while the control was provided with conventional training. Subsequently, Concentration Grid Test (CGT) was used to measure the concentration, and FIBA-LiveStats were collected to evaluate the game statistics and athlete performance. Results. The results showed that there was a significant difference in the effect of NeuroTracker training and conventional training on increasing concentration. Furthermore, there was no significant difference in the effect of NeuroTracker training and conventional training on improving game performance. Conclusion. The practice of NeuroTracker 3D MOT training positively influenced concentration. However, there are no significant differences in contrast to conventional training on game performance.
\end{abstract}

KEYWORDS: NeuroTracker 3D-MOT, Concentration, Game Performances.

\section{INTRODUCTION}

Perceptual-cognitive training is designed to improve an athlete's performance during the search, identification, processing, and integration of information with the knowledge and ability to perform appropriate actions (1) These skills are essential to improve sporting performance, and several methods have been implemented to ensure perfection, including visual simulation, video projection, and the use of Neurotrackers (2). Besides, these approaches are widely used by coaches, sports experts, and athletes to enhance skills (3) and are potentially practiced in diverse sports, including the dynamic team forms (e.g., football, basketball, ice skating, baseball, softball, and field hockey) and individual sports (e.g., running, tennis) $(2,4)$. Moreover, perceptualcognitive training is also applied to improve athlete concentration for the actual game (5).

The act of concentrating is a vital skill in many sports, as observed in basketball (6). These athletes are practically expected to perform better during the game and observe the fast movements

*. Corresponding Author:

Komarudin Komarudin, Ph.D.

E-mail: komarudin_pko@upi.edu 
of fellow team players as well as the opponents. Also, it is essential to understand and interpret information in the court, and also make decisions regarding where the ball is to be passed, or the anticipated position of team players $(7,8)$. The external environment quickly changes during a competition, and significant focus and concentration are required to keep up with the fast-movement of both players and ball. This is possibly improved by athletes' engagement in some training, including through Neurotracker training.

Based on previous studies, training using a 3D Multiple Object Tracking (3D-MOT) NeuroTracker software have the potential to enhance concentration and game performance (9). Besides, related studies have focused on the individuals' observation and response-ability towards various stimuli in a basketball game. The experimental study performed by Romeas, Guldner, and Faubert (2016) showed a significant improvement in precision on ball passing, and not in dribbling or shooting (10). A study conducted by Mangine, et al., (2014) examined the relationship between visual tracking speed (VTS) and reaction time (RT) during basketball game performance. This was measured in terms of the assists (AST), turnovers (TO), assist-to-turnover ratio (AST/TO), and steals (STL) (8). The results showed the existence of an association with VTS and not RT, while the correlation with concentration was not explored. This current study is, therefore, this study aims to examine differences in the effect of NeuroTracker training and conventional training on increasing concentration and game performance of basketball athletes.

\section{MATERIALS AND METHODS}

This experimental study involved pre-and post-training sessions with 21 basketball athletes, and were classified into experimental (6 male, 5 female; Age $19.909 \pm 1.136$ ) and control groups (6 male, 4 female; Age $19.900 \pm 0.738$ ). The investigation was focused on two essential skills, including concentration and game performance, trial based on field goal attempts, free throws, two-point made, three-point shots, number of assists, total defensive, and offensive rebounds, number of fouls, number of turnovers, total steals, number of block shots (BS) FIBA-LiveStats 2018 (11). The experiment was performed 12 times from May 2020 to August 2020, and four trained observers were involved in the observation both pre- and post-training. Furthermore, all participants were informed of the treatments and voluntarily signed the right to be involved. Therefore, both written and oral informed consent was sought.

Data Collection Technique. The Concentration Grid Test (CGT) was created by Haris \& Haris in 1984 (12) and game performance statistics from FIBA-LiveStats (FLS) Version 7 (13), were used to obtain data. Specifically, CGT was applied in mental focus and concentration practices, with a $10 \times 10$ grid characterized by twodigit numbers from 00 to 99 . Furthermore, participants were required to connect the lowest to the highest numbers by placing a check or across, while the scoring was determined by the total numbers in sequence. Moreover, there was a pre- and post-test administration, performed within 60 seconds. The FIBA-LiveStats are provided by game performance data from the International Basketball Federation (FIBA), and also information about other components. These include field goal attempts, free throws, two-point made, three-point shots, number of assists, total defensive, and offensive rebounds, number of fouls, total; turnovers, number of steals, and total block shots.

Procedure. The study involved a Neurotracker, which is a 3-dimensional multiple object tracking (3D MOT) task to improve the athlete's working memory performance and attention. Before the experiment, participants were informed of the procedure and placed in a quiet room to evaluate the level of concentration. Therefore, treatments were provided for 8 minutes based on the available program, and the experimental group was trained for up 12 meetings (three times a week). Besides, a total of 20 CORE sessions were performed during treatments with the Neurotracker 3D-MOT program. The results were assessed, with all participants compelled to follow similar standards and procedures. Also, the individuals were required to sit in front of the computer and wear 3D glasses during each trial, and a 3D transparent cube encompassing eight identical yellow balls displayed on a TV screen was used.

The first phase of the 20 trials was characterized by athletic warm-ups. This required the candidates to sit in front of the screen and were asked to observe eight identical yellow balls present. The balls tend to move simultaneously through a specified path. Before the movement, a total of 
four balls exhibited a color change and were used as the tracking target. These distinct balls changed back to yellow during movement, and the athletes were asked to track the position of all four in eight seconds, and also when stationary, by clicking the respectively inscribed numbers. The movement speed depended on the scores made by the athletes in the previous session. Figure 1 shows an increase in speed on instances where the athletes possess a successful identification characteristic and vice versa. Moreover, those in the control groups performed a routine exercise three times a week at the basketball club without any NeuroTracker exercise.

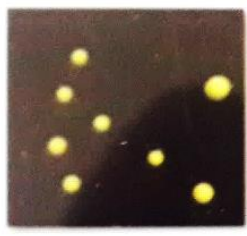

a

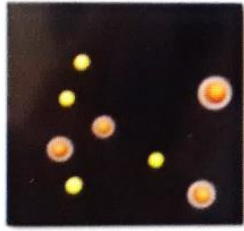

b

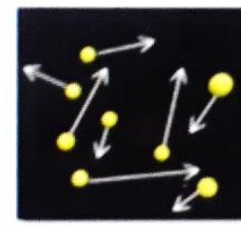

C

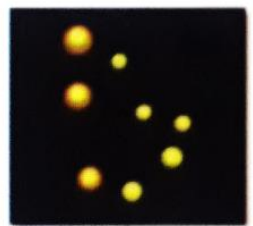

d

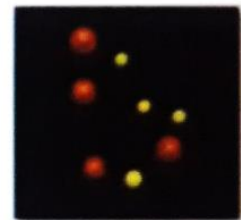

e

Figure 1. Steps in Neurotracker Training (Adapted, Thomas Romeas, Antoine Guldner, Jocelyn Faubert, 2016)

Data Analysis Technique. This study used ANCOVA analysis was the post-test score was the dependent variable, the group was the fixed factor, and the pre-test was the covariate. Data analysis used the SPSS version 26 program.

The parametric statistics were used to evaluate the information on concentration and performance variables. See Figure 1. Therefore, an independent t-test was used to determine differences in the effect of the NeuroTracker 3D MOT and conventional training on the increasing values on concentration, and game performance. Meanwhile, ANCOVA was used to evaluate the differences in the effects of the NeuroTracker 3D MOT compared to conventional training, and data analysis involved the SPSS version 21.0 program.

\section{RESULTS}

Before doing ANCOVA analysis, several assumptions or conditions must be met, namely normality test, homogeneity of variance, homogeneity of regression (slope), and linearity test. Table 1 shows the results of the ANCOVA assumption test on the concentration and game performance variables.

Table 1. ANCOVA Assumptions Test Results on Concentration and Game Performance Variables

\begin{tabular}{|c|c|c|}
\hline \multicolumn{3}{|c|}{ Concentration } \\
\hline Assumptions Test & $\mathbf{p}$ & Information \\
\hline \multicolumn{3}{|l|}{ Normality test pretest_Concentration } \\
\hline Neurotracker_group & 0.134 & Assumption are met \\
\hline Conventional_group & 0.228 & Assumption are met \\
\hline \multicolumn{3}{|l|}{ Postest_Concentration } \\
\hline Neurotracker_group & 0.448 & Assumption are me \\
\hline Conventional_group & 0.202 & Assumption are met \\
\hline Homogeneity of variance & 0.338 & Assumption are met \\
\hline Homogeneity of Regression (Slope) & 0.425 & Assumption are met \\
\hline Linearity test & 0.035 & Assumption are met \\
\hline \multicolumn{3}{|l|}{ Normality test pretest_game performance } \\
\hline Neurotracker_group & 0.050 & Assumption are met \\
\hline Conventional_group & 0.537 & Assumption are met \\
\hline \multicolumn{3}{|l|}{ Postest_game performance } \\
\hline Neurotracker_group & 0.068 & Assumption are met \\
\hline Conventional_group & 0.073 & Assumption are me \\
\hline Homogeneity of variance & 0.926 & Assumption are met \\
\hline Homogeneity of Regression (Slope) & 0.644 & Assumption are met \\
\hline Linearity test & 0.045 & Assumption are met \\
\hline
\end{tabular}


Table 1 shows that in the concentration and performance game variables all the assumptions of the ANCOVA test are met, so this study can use the ANCOVA test. To see an illustration of whether there is a difference in the average concentration score and game performance after being given the exercise (posttest in each exercise), as shown in Table 2.

Table 2. shows that the posttest scores on concentration and game performance descriptively can be concluded that the average concentration and game performance scores in the NeuroTracker group are higher than the conventional group. The next analysis was continued by testing whether there was a significant difference in the average score of concentration and game performance in the NeuroTracker and conventional training groups. The analysis results can be seen in Table 3.

Table 2. Posttest Score on Concentration and Game Performance

\begin{tabular}{lccc}
\hline Variables & N & Mean & SD \\
\hline Concentration & & & \\
$\quad$ NeuroTracker & 11 & 17,91 & 1,00 \\
$\quad$ Conventional & 10 & 12,70 & 1,50 \\
Game performance & & 16,64 & 4,10 \\
$\quad$ NeuroTracker & 11 & 13,80 & 1,50 \\
$\quad$ Conventional & 10 & & \\
\hline
\end{tabular}

Table 3. Analysis of Differences in Mean Concentration and Game Performance Scores Based on the Neurotracker Exercise Group and Conventional Exercise.

\begin{tabular}{lccccc}
\hline Variable & B & SE & P-value (Bonferroni test) & $\begin{array}{c}\text { p-value (Parameter } \\
\text { estimates) }\end{array}$ & 95\% CI \\
\hline Concentrartion & 5,258 & 0,814 & 0,001 & 0,001 & $3,547-6,969$ \\
Game performance & 2.091 & 2.702 & 0,449 & 0,449 & $3,547-6,969$ \\
\hline
\end{tabular}

Table 4. Results of NeuroTracker 3D MOT training

\begin{tabular}{|c|c|c|}
\hline \multirow{2}{*}{ Subjects } & \multicolumn{2}{|c|}{ Means } \\
\hline & Initial Baseline & Current Baseline \\
\hline $\mathbf{A}$ & 0.69 & 0.74 \\
\hline B & 1.77 & 2.46 \\
\hline $\mathrm{C}$ & 0.97 & 1.77 \\
\hline D & 0.52 & 1.13 \\
\hline $\mathbf{E}$ & 1.78 & 2.49 \\
\hline $\mathbf{F}$ & 1.12 & 2.30 \\
\hline G & 0.46 & 1.25 \\
\hline $\mathbf{H}$ & 1.16 & 1.80 \\
\hline I & 0.63 & 1.16 \\
\hline $\mathbf{J}$ & 0.93 & 1.41 \\
\hline K & 1.66 & 2.08 \\
\hline
\end{tabular}

The value of treatment variables in the concentration using Bonferroni adjustment obtained p-value $0.001<0.05$, so it can be concluded that there is a difference in the average concentration score of athletes trained by NeuroTracker with conventional training. On the parameter estimates, it can be seen that Nurotracker training was effective in increasing the athlete's concentration ( $\mathrm{P}$-value $0.001 ; \mathrm{B}=$ 5.258; $\mathrm{SE}=0.814)$. Furthermore, in the performance game using Bonferroni adjustment, the $p$-value is $0.449>0.05$, so it can be concluded that there are significant differences in performance games trained using NeuroTracker 3D MOT and conventional. On parameter estimates, it can be seen that NuroTracker training is not effective in improving the game performance of basketball athletes (P-value 0.449 $<0.05 ; \mathrm{B}=2.091 ; \mathrm{SE}=2.702)$. Table 4 shows the results of the NeuroTracker 3D MOT training treatment on 11 subjects for 12 sessions. The result shows an increase in the individuals' ability to perform object tracking, which commences at the Initial to Current Baseline. This indicates the acquisition of focus abilities, particularly in the aspect of object identification. 


\section{DISCUSSION}

The results of statistical analysis showed significant differences in athlete concentration between groups treated with NeuroTracker and conventional training. However, the effects were not substantial in the aspect of game performance. Particularly, NeuroTracker 3D-MOT training is considered a perceptual-cognitive training program (14) widely used to increase the biological motion perception required by athletes to improve sporting performance. The training is developed to enhance the individuals' dynamic information scanning process, reflect several fundamental aspects, and the concentration demand levels required. Also, this is crucial for athletes to perform the complex and useful movements needed to maintain a working memory and promote concentration (15). Hence, this study proves the ability of NeuroTracker 3DMOT training to significantly increase concentration amongst basketball athletes.

The treatment strives to combine several key elements, including awareness and focus to perform simple but challenging tasks. Therefore, athletes during training are expected to carefully consider four complex and dynamic targets (objects) and simultaneously maintain concentration as well as a focus before attaining high-speed movements (16). This process enhances individual awareness as well as concentration and is estimated to ensure quick and precise decisions making, required to provide significant improvements. Besides, the phenomenon is observed especially in the aspect of attention, memory, information processing speed, and learning (17).

Based on the neuroscience perspective, NeuroTracker 3D-MOT training is estimated to utilize a modern neuroplasticity principle. This condition indicates the ease of human brain adaptability to environmental demands through functions of reorganization, increasing efficiency, and new neural network growth. This organ has the potential to adapt through self-modification required for better response to performance (18). Furthermore, it is essential to task the brain for increased awareness through MOT, to ensure stimulation. This is a perceptual skill, where athletes are expected to distribute attention and concentration on several points in the environment. Therefore, these abilities are very important in a basketball game because athletes are expected to move dynamically, and good decisions are possibly made after careful observation of all field movements, including those of team members, opponents, and the ball (19).

Similarly, Zhang, et al., (2019) reported the following requirements for basketball athletes to achieve a successful performance: read the opponents' play, engage in good movements and predict the opponent's advances, identify game patterns, and process biological basic movement (20). The NeuroTracker training is assumed to improve athlete concentration, which is a strength in basketball adopted to focus on mental resources and maintain awareness during games. Therefore, the absence indicates loss of athlete awareness (16) as concentration is considered an essential ability estimated to greatly influence the decision-making process. Hence, long term concentration using MOT is very important in basketball (21). Meanwhile, increased tracking speed threshold against objects is considered the most important aspect of NeuroTracker treatment, as faster movements require greater concentration, while the focus is necessitated with higher speeds (22).

Based on the neurological perspective, NeuroTracker 3D-MOT training plays a significant role in facilitating concentration, visual information processing, and enhancing the working memory (23). The imagery training study showed the exercise ability to ensure the high-level activation of several brain parts while concentrating (24). Specifically, the parietal, frontal, and cortex parts have the potential to increase athlete concentration and eye movement, while the middle temporal brain is involved in perceiving the motion to perform during the MOT process. The act of reading the opponent's play pattern indicates intellectual involvement, which the athlete perceives in the motion or action process by activating both the dorsal and ventral pathways (25). Also, there is a need for cognitive resources on the multiple targets process, particularly on instances of similar disturbances during the process (26). Based on neuroimaging studies, the activation process of subjects performing MOT exercise tasks is demonstrated along with the dorsal attention network, including the frontal eye fields, superior parietal lobe, anterior, and posterior intraparietal sulcus. However, deactivation is observed within the dorsal medial network, comprising the medial prefrontal and temporal cortex (27). Moreover, 
the segment of the dorsal attention network involved in goal-driven (top-down) selections and responses tend to strongly support the selective and sustainable visuospatial attention (28).

This present study acknowledges the absence of any significant differences in the playing performance of basketball athletes between the groups exposed to NeuroTracker 3D MOT and conventional training. Therefore, game performance was based on a real context, as observed in a match. The participants from both groups competed and the individual performances were evaluated using FIBA-LiveStats. Meanwhile, a professional assessment team measured the game performance, and the team's maximum functioning was achieved in instances where abilities and excellence are adequately maintained, and also the prestige appreciated by other teams after a victory. The maximum performance is supported by physiological as well as psychological abilities, including commitment, control, challenge, and coping skills (19). These are further considered to be very important in achieving match success, as psychological abilities aim to maximize all player's potentials obtained during training. Besides, athletes ought to possess the ability to display these potentials during competition.

These psychological abilities are closely related to the athletes' emotional condition, as emotions play a crucial role under match situations, and influence good or bad performance, as well as win or loss (29). Furthermore (30) reported on the close relationship between emotion and concentration, where happiness provided positive energy and consequently improved the game outcome. This resulted from the ability to predict the attention sources required to achieve goals. Hence, the concentration attributed to the designated tasks is possibly externalized (31, 32). Conversely, negative emotions including anxiety, frustration, and anger instigate a decline in performance, due to the intense feeling of tension and absence of focus.

Besides, self-confidence is considered a basic capital needed to achieve success in athlete performance, despite being in a physiologically anxious state. Therefore, high levels alongside low somatic anxiety are implicated in greater concentration and consequently higher accomplishments (7) id various failures (33). However, numerous studies have emphasized the tendency for anxiety to impair athlete attention and concentration. Therefore, the optimal psychological condition is required to improve performance during training processes or in competitions. This requires the dominance of positive emotions, as well as substantial awareness and arousal, to ensure a sustained focus on the tasks. The absence of concentration in a basketball match increases the tendency for mistakes and consequently impacts team performance.

The results showed no significant difference between the groups treated with NeuroTracker 3D MOT and conventional training in the aspect of game performance. This was attributed to athlete exposure to complex influencing conditions. Also, athletes are expected to deal with unique match environments during the game, including the aspect of spectators, referees, officials, weather, teammates, and the quality of the opposing team's performance. Moreover, wins and losses are largely dependent on several situational variables described in the match statistics, including two-and-three point field goals (34), free throws (35), offensive and defensive rebounds, blocked shots (20), turnovers, steals, personal fouls (4), and assists (36). The NeuroTracker training was estimated to have influenced athletes' focus on executing specified tasks during every game, which further predicts wins and losses.

The basketball game performance is shown in match statistics and is subsequently used by coaches to optimize training patterns and load, alongside decision making. Furthermore, the information obtained helps to easily determine the appropriate strategy required to improve team operations (20). According to Simons, et al., (2016), the process and results are also influenced by the quality and the type of opponent, and more offensive counterparts provide better performances (37). This factor also influences victory and is often determined during the second and third quarters of a match. The effect of team quality is recognized on all variables except in the aspect of free throws, offensive rebounds, and errors in the Spanish professional basketball league. However, this is not the case with professional athletes, as explained in the study by Zhang, et al., (2019) where the best team in the NBA league is not influenced by the game location or opponent quality (20). Therefore, NBA athletes are believed to already have 
numerous abilities above average compared to other teams. This phenomenon is also attributed to the individual psychological strength, which enables the demonstration of best performances, despite the in-game challenges faced.

Furthermore, players and teams always show unique traits while playing with different opponents at different times $(2,38)$. This study is highly relevant in the conditions of this study subject, and different opponents, as well as game quality, were determined to influence athlete play performance. The results of this study reinforce the above statement that the mastery of basketball technique skills when playing such as field goals, mean pretest and posttest experiment and control, (mean pre 40.82, post 26.00) and (mean pre 20.70, post 37.38 ), shooting 2 points (mean pre 48.72-post 27.55 and mean pre 21.88 , post 28.13 ), 3 points (mean pre 3.03 , post 4.55 and (mean pre 0.13 , post 28.13), and free throws (mean pre 18.18, post 33.73 ) and (mean pre 5.00, post 6.25). which are a sub of performance game variables there is no significant difference in these variables as a whole in the NeuroTracker trained group and the conventional trained group ( $p>$ $0.05)$. However, the ability of free throws during the initial game test shows that there is a difference between the NeuroTracker group and the conventional group ( $\mathrm{p}<0.05$ ), but in this condition, the subject has not received any treatment from the researchers. The absence of differences in the influence of these two forms of training on performance games is due to many influencing factors, especially those related to the quality of athletes and opponents. When playing, the mastery of the basketball technique will be different from not playing because there are no factors that influence it. Besides, the subjects trained with NeuroTracker and conventionally turned out to be a lot of fouls, both personal fouls (for experiment mean pre 1.27, post 1.09 and (control mean pre 1.63, post 1.38), and foul down (for experiment mean pre 0.91 , post 2.30) and control (mean pre 1.75post 0.88 ). This situation will affect the quality of the athlete's performance, especially in the games.

Based on psychological assessment, athletes are challenged by the event of adapting to new environments and varied competitive situations. Therefore, coaches ought to cautiously improve the psychological quality and enhance the focus required for proper participation in any situation. This is achieved by adopting NeuroTracker training at every drill session, because of the ability to increase concentration and basketball game performance. The participating individuals also influence the team activity, and interaction between players significantly affects quality improvement. However, the result of this present study indicates the absence of any significant differences between the average score of game performance between the experimental and control groups. This variable is, therefore, assumed to be greatly influenced by numerous factors, including the match environment, opponent quality, the location of play, as well as the integrity of the individuals in a team. Also, other psychological aspects are considered as important parts of the training program.

\section{CONCLUSION}

Based on the results of treatment given to subjects who are university athletes for 12 meetings, this study concludes that there was a significant difference in the effect of NeuroTracker training and conventional training on increasing concentration. Furthermore, there was no significant difference in the effect of NeuroTracker training and conventional training on improving the game performance of basketball athletes. Based on the average gain score, NeuroTracker training is better than conventional training to improve concentration and game performance for basketball athletes. Therefore, in the training process, NeuroTracker is recommended to be used to increase athlete concentration. However, both forms of training can be used to improve game performance, and many factors influence performance improvement, including the ability and quality of the opponent. This study has limitations; including First, the NeuroTracker training program still adopts the CORE Session, so the recommendation for future researchers to use the CORE + Mode program or the next program in NeuroTracker. Second: the number of subjects is limited to be researched, and athletes' achievement is only at the university level so that further researchers need to use a larger subject with elite athletes. Furthermore, manual measurement, especially for measuring concentration, should use a measuring device capable of capturing the full ability of the brain, such as the Electroencephalogram (EEG). 


\section{APPLICABLE REMARKS}

- The training process requires a science and technology approach, as well as the support of the right methods to produce peak performance.

- It is hoped that the results of this research adaptable for coaches, athletes, and physical education teachers in increasing the concentration of athletes so that athletes stay focused and able to anticipate, and possess the ability to make decisions quickly and accurately.

\section{REFERENCES}

1. Marteniuk RG. Cognitive information processes in motor short-term memory and movement production. In Motor control. Acad Press. 1976:175-186. doi: 10.1016/B978-0-12-665950-4.50012-2

2. Stiles TTMFHM. The effects of perceptual-cognitive training with NeuroTracker on executive brain functions among elite athletes. Cogent Psychol. 2018.;5:1544105. doi: 10.1080/23311908.2018.1544105

3. Fadde PJ. Interactive Video Training of Perceptual Decision-Making in the Sport of Baseball, Technical Instruction, Cognition and Learning. Cogn Learn. 2006;4:265-285.

4. Leicht AS, Gómez MA, Woods CT. Explaining match outcome during the men's basketball tournament at the Olympic Games. J Sports Sci Med. 2017;16(4):468-473.

5. Renshaw I, Davids K, Araujo D, Lucas A, Roberts WM, Newcombe DJ, et al. Evaluating Weaknesses of "Perceptual-Cognitive Training" and "Brain Training" Methods in Sport: An Ecological Dynamics Critique. Front Psychol. 2018;9:2468. doi: 10.3389/fpsyg.2018.02468 pmid: 30719015

6. Angel Gomez M, Lorenzo A, Sampaio J, Jose Ibanez S, Ortega E. Game-related statistics that discriminated winning and losing teams from the Spanish men's professional basketball teams. Coll Antropol. 2008;32(2):451-456.

7. Eysenck MW, Calvo MG. Anxiety and performance: The processing efficiency theory. Cogn Emot. 1992;6:409-434. doi: 10.1080/02699939208409696

8. Mangine GT, Hoffman JR, Wells AJ, Gonzalez AM, Rogowski JP, Townsend JR, et al. Visual tracking speed is related to basketball-specific measures of performance in NBA players. $J$ Strength Cond Res. 2014;28(9):2406-2414. doi: 10.1519/JSC.0000000000000550 pmid: 24875429

9. Appelbaum LG, Erickson G. Sports vision training: A review of the state-of-the-art in digital training techniques. Rev Sport Exerc Psychol. 2018;11(1):160-189. doi: 10.1080/1750984X.2016.1266376

10. Romeas T, Guldner A, Faubert J. 3D-Multiple Object Tracking training task improves passing decisionmaking accuracy in soccer players. Psychol Sport Exerc. 2016;22:1-9. doi: 10.1016/j.psychsport.2015.06.002

11. Krešimir L, Horvat T, Havaš LI. Information System for Performance Evaluation in Team Sports. Int $J$ Bus Manag Technol. 2018;2(1):53-59.

12.Lane AM, Terry PC, Beedie CJ, Stevens M. Mood and concentration grid performance: Effects of depressed mood. Int J Sport Exerc Psychol. 2004;2(2):133-145. doi: 10.1080/1612197X.2004.9671737

13.Group GS. Fiba livestats (FLS) Version 7 [Internet]. 2019. Available from: http://www.fibaorganizer.com/.

14.Legault I, Allard R, Faubert J. Healthy older observers show equivalent perceptual-cognitive training benefits to young adults for multiple object tracking. Front Psychol. 2013;4:323. doi: 10.3389/fpsyg.2013.00323 pmid: 23761025

15.Faubert J, Sidebottom L. Perceptual-cognitive training of athletes. J Clin Sport Psychol. 2012;6(1):85102. doi: $10.1123 /$ jesp.6.1.85

16. Faubert J. Professional athletes have extraordinary skills for rapidly learning complex and neutral dynamic visual scenes. Sci Rep. 2013;3:1154. doi: 10.1038/srep01154 pmid: 23378899

17. Assed MM, Rocca CCA, Garcia YM, Khafif TC, Belizario GO, Toschi-Dias E, et al. Memory training combined with 3D visuospatial stimulus improves cognitive performance in the elderly: pilot study. Dement Neuropsychol. 2020;14(3):290-299. doi: 10.1590/1980-57642020dn14-030010 pmid: 32973982

18. Kolb J, Beauchamp P, Faubert J. Visual perception training: cutting edge psychophysics and 3D technology applied to sport science. CIRCuit. 2011:1-14. 
19.Ramzi S, Besharat MA. The impact of hardiness on sport achievement and mental health. ProcediaSocial Behav Sci. 2010;5:823-826. doi: 10.1016/j.sbspro.2010.07.192

20.Zhang S, Lorenzo A, Zhou C, Cui Y, Gonçalves B, Angel Gómez MP. Performance profiles and opposition interaction during game-play in elite basketball: evidences from National Basketball Association. Int J Perform Anal Sport. 2019;19(1):28-48. doi: 10.1080/24748668.2018.1555738

21. Memmert DP. Pay attention! A review of visual attentional expertise in sport. International Review of Sport and Exercise Psychology. Int Rev Sport Exerc Psychol. 2009;2(2):119-138. doi: 10.1080/17509840802641372

22.Feria CS. The effects of distractors in multiple object tracking are modulated by the similarity of distractor and target features. Perception. 2012;41(3):287-304. doi: 10.1068/p7053 pmid: 22808583

23.Parsons B, Magill T, Boucher A, Zhang M, Zogbo K, Berube S, et al. Enhancing Cognitive Function Using Perceptual-Cognitive Training. Clin EEG Neurosci. 2016;47(1):37-47. doi: 10.1177/1550059414563746 pmid: 25550444

24. Howe PD, Horowitz TS, Morocz IA, Wolfe J, Livingstone MS. Using fMRI to distinguish components of the multiple object tracking task. J Vis. 2009;9(4):10 11-11. doi: 10.1167/9.4.10 pmid: 19757919

25.Goodale M, A., ADM. Separate visual pathways for perception and action. 1992:20-25. doi: 10.1016/0166-2236(92)90344-8

26. Dorum ES, Alnaes D, Kaufmann T, Richard G, Lund MJ, Tonnesen S, et al. Age-related differences in brain network activation and co-activation during multiple object tracking. Brain Behav. 2016;6(11):e00533. doi: 10.1002/brb3.533 pmid: 27843692

27. Alnaes D, Kaufmann T, Richard G, Duff EP, Sneve MH, Endestad T, et al. Attentional load modulates large-scale functional brain connectivity beyond the core attention networks. Neuroimage. 2015;109:260-272. doi: 10.1016/j.neuroimage.2015.01.026 pmid: 25595500

28. Corbetta M, Patel G, Shulman GL. The reorienting system of the human brain: from environment to theory of mind. Neuron. 2008;58(3):306-324. doi: 10.1016/j.neuron.2008.04.017 pmid: 18466742

29. Krane V, Williams JM. Psychological characteristics of peak performance. Appl Sport Psychol Personal Growth Peak Performance. 2006;5:207-227.

30. Vast RL, Young RL, Thomas PR. Emotions in sport: Perceived effects on attention, concentration, and performance. Aust Psychol. 2010;45(2):132-140. doi: 10.1080/00050060903261538

31. Carver CS, Scheier MF. Origins and functions of positive and negative affect: A control-process view. Psychol Rev. 1990;97(1):19-35. doi: 10.1037/0033-295X.97.1.19

32.Fredrickson BL. The role of positive emotions in positive psychology. The broaden-and-build theory of positive emotions. Am Psychol. 2001;56(3):218-226. doi: 10.1037//0003-066x.56.3.218 pmid: 11315248

33.Rusk N, Maya TFR. "Performance and learning goals for emotion regulation.". Motiv Emot 354. 2011:444-460. doi: 10.1007/s11031-011-9229-6

34. Çene E. What is the difference between a winning and a losing team: insights from Euroleague basketball. Int J Perform Anal Sport. 2018;18(1):55-68. doi: 10.1080/24748668.2018.1446234

35. Conte D, Lukonaitiene I. Scoring Strategies Differentiating between Winning and Losing Teams during FIBA EuroBasket Women 2017. Sports (Basel). 2018;6(2). doi: 10.3390/sports6020050 pmid: 29910354

36.Puente C, Coso JD, Salinero JJ, Abián-Vicén J. Basketball performance indicators during the ACB regular season from 2003 to 2013. Int J Perform Anal Sport. 2015;15(3):935-948. doi: $10.1080 / 24748668.2015 .11868842$

37. Simons DJ, Boot WR, Charness N, Gathercole SE, Chabris CF, Hambrick DZ, et al. Do "Brain-Training" Programs Work? Psychol Sci Public Interest. 2016;17(3):103-186. doi: 10.1177/1529100616661983 pmid: 27697851

38. McGarry T, Anderson DI, Wallace SA, Hughes MD, Franks IM. Sport competition as a dynamical selforganizing system. J Sports Sci. 2002;20(10):771-781. doi: 10.1080/026404102320675620 pmid: 12363294 